\title{
EDITORIAL \\ Rupture risk of unruptured aneurysms: beyond size and shape
}

\author{
Eric C. Peterson, MD, and Roberto C. Heros, MD \\ Department of Neurological Surgery, University of Miami Miller School of Medicine/Jackson Memorial Hospital, Miami, Florida
}

$\mathrm{T}$ HIS issue of the Journal of Neurosurgery includes the report of a very good study, "Assessment of image-derived risk factors for natural course of unruptured cerebral aneurysms" by Ramachandran and colleagues. ${ }^{1}$ The authors prospectively evaluated 198 unruptured aneurysms from 4 institutions that were managed conservatively. The aneurysms were carefully studied with image-derived morphological, volumetric, and flow dynamic parameters. The patients were then prospectively followed for a median time of approximately 22 months and evidence of growth and/or rupture was recorded. The aneurysms were divided into a "stable" group (no significant growth or rupture) and an "unstable" group (evidence of growth and/or rupture). None of the aneurysms ruptured during the follow-up period, $10 \%$ grew, and $75 \%$ were stable (15\% were lost during follow-up). Statistically, there was no difference in any of the recorded metrics between the stable and the unstable groups.

We were impressed with this article because, to our knowledge, it is the first attempt specifically designed to prospectively collect this type of data and see if it could be correlated with growth and/or rupture during followup. By contrast, there have been a multitude of reports of elegant studies analyzing parameters of this type in aneurysms that present with rupture and comparing them with aneurysms that are discovered in an unruptured state. Clearly, significant differences have been found in these parameters in the group of ruptured aneurysms as compared to the unruptured aneurysms. The implication in most of these studies has been that when unruptured aneurysms are found to have characteristics that are more frequently seen in ruptured aneurysms, they are more likely to rupture in the future, and therefore a stronger rationale to treat unruptured aneurysms with those characteristics is suggested. It certainly would be nice if it could be confirmed, in prospective studies of the type performed by Ramachandran and colleagues, that indeed unruptured aneurysms with characteristics more typical of ruptured aneurysms have a higher risk of rupture in the future. This certainly would help us to refine our criteria as to when unruptured aneurysms should be treated and when not. Unfortunately, this particular study lends no support to the hypothesis that morphological, volumetric, and flow dynamic characteristics of unruptured aneurysms can be used to predict which of these aneurysms are more likely to either grow or rupture. We think that using growth as an end point is justified because it is widely accepted (although not definitively proven as far as we know) that aneurysmal growth is a strong predictor of rupture.

If there indeed were to be no particular morphological features predictive of rupture in an unruptured aneurysm, one would have to ask why is it that when we compare ruptured aneurysms with unruptured aneurysms such differences consistently exist. The most obvious explanation is that aneurysms change upon rupture. Of course, this is not a new concept, and this has been given repeatedly as a likely explanation for differences in morphology between ruptured and unruptured aneurysms. There obviously is more to it than just a change in morphology caused by the rupture, but that this is a factor is, in our opinion, a very strong likelihood. We recently had an experience with a patient who presented with a diffuse subarachnoid hemorrhage (SAH) and multiple aneurysms. Based on size and irregularity, we (almost certainly wrongly) attributed the SAH to the middle cerebral artery aneurysm that we then proceeded to treat endovascularly. One week after discharge, the patient came back with a rehemorrhage from an untreated small pericallosal artery aneurysm that had a very regular shape on initial angiography. The repeat angiogram showed that this aneurysm had changed dramatically both in shape and in size, which we attributed to the probable development of a pseudoaneurysm at the site of rupture (Fig. 1).

One of the major strengths of the article by Ramach- 


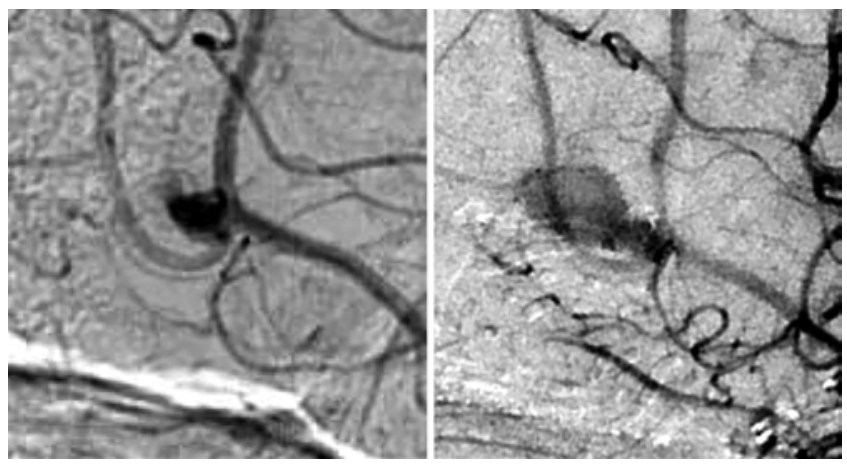

FIG. 1. Lateral catheter angiogram of internal carotid artery injection obtained at time of initial hemorrhage (left) and at time of rehemorrhage (right).

andran and colleagues is their thorough and thoughtful discussion of the limitations of the study. They repeatedly emphasize, and they are very careful in their wording, that their study's findings "do not support the hypothesis that image-derived metrics can predict aneurysm growth in patients who have been selected for observation...." Their study does not tell us that there are not image-derived metrics that may be predictive of future growth and rupture. If there were such metrics, it is left to future prospective studies, with a greater number of patients and longer follow-up, to show such correlations.

The most obvious limitation of the study, amply discussed by the authors, is the almost certain selection bias that occurred, since it is most likely that aneurysms with certain features that the clinicians believed may be predictive of a higher risk of rupture were treated rather than observed. Implicit in that probable bias is the fact that the aneurysms that were chosen for observation and included in their study were mostly small aneurysms, because the larger aneurysms are much more likely to have been selected for treatment. Clearly, the small aneurysms included in this study are known to have a very benign natural history, with a risk of bleeding that is certainly below $1 \%$ per year, and therefore one would not have expected to have seen many ruptures during a median 2-year followup period. Not surprisingly, the authors found no ruptures. The $10 \%$ incidence of growth, even with this series of relatively small aneurysms, is impressive and it is disappointing that no distinguishing metrics were found for this group of aneurysms that grew. Perhaps with a longer follow-up, which I hope the authors are planning to conduct, differences will emerge.

We want to avoid the usual cliché of suggesting a randomized study to address this question. At this point we already have strong biases, some based on substantial prospective data, about which unruptured aneurysms are more likely to rupture in the future. It is unlikely that we would randomize larger aneurysms, aneurysms with a daughter blister, aneurysms that have shown growth, or aneurysms in patients with a strong family history of these lesions; these are all likely to be treated if possible. Ethically, it would be unacceptable to ignore these biases, which are based on substantial data, although not proven by prospective randomized studies. Therefore, if such a study were to be designed, we would end up randomizing only patients very similar to the ones who have been included in the present study, and we would be unlikely to end at a much different point than where we are at this time.

We certainly need to continue to study the question of whether image-derived metrics can be useful in predicting which unruptured aneurysms have a greater risk of rupture. Clearly, the fact that small unruptured aneurysms are very unlikely to rupture when followed prospectively is in great contrast with the observation that many ruptured aneurysms are very small at the time of rupture. This indicates that there is a dynamic nature to these lesions and at present we have a poor understanding of the factors underlying this dynamism. We need to continue to study not only morphological features, but also the molecular inflammatory mechanisms that may influence this dynamic process of aneurysm development, growth, and rupture.

We heartily congratulate the authors on a very well designed, executed, and reported study.

http://thejns.org/doi/abs/10.3171/2015.4.JNS15465

\section{Reference}

1. Ramachandran M, Retarekar R, Raghavan ML, Berkowitz B, Dickerhoff B, Correa T, et al: Assessment of imagederived risk factors for natural course of unruptured cerebral aneurysms. J Neurosurg [epub ahead of print September 18, 2015. DOI: 10.3171/2015.2.JNS142265]

\section{Disclosure}

The authors report no conflict of interest.

\section{Response}

\section{Robert Harbaugh, MD}

Department of Neurosurgery, Penn State University, Hershey, Pennsylvania

I am grateful to Drs. Peterson and Heros for their insightful and generous review of our paper "Assessment of image-derived risk factors for natural course of unruptured cerebral aneurysms." I find nothing in their thoughtful analysis with which I disagree. As noted in the editorial-and in our paper-it is important to stress the limitations of this study and how we can move forward from here.

I believe that a selection bias exists in our study population that mimics a selection bias in other studies of unruptured aneurysms. ${ }^{2}$ Physicians with expertise in the management of patients with unruptured intracranial aneurysms are more likely to recommend invasive treatment for aneurysms with larger size and irregular shape. In this study of patients with unruptured aneurysms, for whom observation rather than invasive treatment was recommended, the nonsphericity index (NSI), a metric that captures the deviation from spherical shape of the aneurysm sac, was $0.11 \pm 0.06$ (mean $\pm \mathrm{SD}$ ). A perfectly hemispherical sac will have an NSI of 0 , and the NSI increases up to a maximum of 1 as the aneurysm becomes more irregular. In earlier reports of patients with unruptured aneurysms, for whom invasive treatment was recommended, all had mean NSI values greater than those in our study. In addi- 
tion, the aneurysms in this study were, on average, smaller than those in earlier reports. By looking only at the aneurysms for which observation was recommended we may have already excluded most aneurysms with a propensity to grow and rupture. A separate study is presently underway to determine if the aneurysms for which invasive treatment was recommended had geometrical indices that were significantly different from the indices of aneurysms for which observation was recommended.

I also agree with Drs. Peterson and Heros that a randomized study of this issue is impractical and would probably give us unreliable information. Clinicians evaluating patients with unruptured aneurysms do not have equipoise in regard to treatment versus observation. Patients thought to be at higher risk of rupture would be treated outside the trial. Patients thought to have a low risk of rupture would be randomized. Such a trial would almost certainly show that observation was preferable and, because this kind of study would be classified as "Level I evidence," we would no longer be able to offer invasive treatment to those patients who might actually benefit. I believe there is a better way to address this question.

Most investigators recognize that in the ideal situation randomized controlled trials (RCTs) let us make causal inferences in a way that no other study design can. Fewer recognize the particular problems with surgical RCTs with regard to intention to treat versus "as treated" analyses, lack of masking, lack of equipoise in those recommending treatment and the effect this has on the study population, the variability of surgical skill and judgment, the intermittent nature of RCTs when invasive and noninvasive therapies are changing, the duration of the study for prophylactic surgical procedures, and how all of these factors affect the generalizability and practitioner acceptance of RCT results.

I believe there is a better option than the RCT for certain problems such as management of unruptured aneurysms. One can design a registry in such a way that reliable causal inferences can be made using propensity analysis. ${ }^{1}$ The questions we need to address to create a registry that would allow us to make causal inferences about treatment options for unruptured aneurysms would include the following:
1. What is the hypothesis we are testing (i.e., what randomized study do we want to model)? In this case the hypothesis might be "Invasive treatment of unruptured aneurysms improves the functional health status of patients compared to observation alone."

2. Who are the decision makers? The patient, evaluating physicians, and family members are the decision makers.

3. What are the key covariates that are used for treatment assignment? These would include the following: 1) patient-specific factors such as patient age, prior aneurysm rupture, medical comorbidities, social history, family history, and patient preferences; 2) aneurysm-specific factors such as aneurysm size, shape, and location; and 3) physician-specific factors such as whether the physician making the recommendation is an endovascular specialist, an open surgical specialist, both, or neither, and the years of experience of the physician and his or her practice setting.

4. Can we quantify the covariates? Yes.

5. What clinically meaningful outcomes do we want to measure? Mortality rate, aneurysmal SAH, and functional health status should be measured.

6 . What sample size will be necessary? This can be calculated based on available data from previous registries and other studies.

7. How long would the registry need to run? This can also be calculated based on available data.

8. What established registry infrastructure is available? The NeuroPoint Alliance is available.

If we create such a registry we will be able to make causal inferences regarding the best treatment options for patients with unruptured intracranial aneurysms that would be more reliable that the causal inferences we could make from a nongeneralizable RCT.

\section{References}

1. Rubin DB: For objective causal inference, design trumps analysis. Ann Appl Stat 2: 808-840, 2008

2. Wiebers DO, Whisnant JP, Huston J, Meissner I, Brown RD, Piepgras DG, et al: Unruptured intracranial aneurysms: natural history, clinical outcome, and risks of surgical and endovascular treatment. Lancet 362:103-110, 2003 